\title{
Alternated Desorption-absorption Accelerated Aging of Salix psammophila Sand Barrier
}

\author{
Rui-Dong Wang, ${ }^{\text {a, }}$ Xiao-Hong Dang, ${ }^{\text {a,b }, \dagger}$ Yong Gao, ${ }^{\text {a,* }}$ Xia Yang, ${ }^{\text {a }}$ Yu-Mei Liang, ${ }^{\text {a }}$ Shuai \\ $\mathrm{Qi}^{\mathrm{a}}{ }^{\mathrm{a}}$ and Chen Zhao ${ }^{\mathrm{a}}$
}

\begin{abstract}
Salix psammophila has been extensively used as a sand barrier material in many desertification control applications. Thus, understanding its degradation processes with long-term environmental moisture changes is essential. In this paper, via alternated desorption-absorption treatment of the S. psammophila sand barrier, damage of differing degrees occurred, and moisture variation was simulated. Through FTIR, X-ray diffraction, SEM, and other characterization methods, changes in macroscopic morphology and physical-mechanical properties of S. psammophila sand barrier were tracked, evaluated, and compared, and the causes were analyzed. The results showed that the alternated desorption-absorption accelerated aging treatment weakened the physical-mechanical properties of the $S$. psammophila sand barrier. The microscopic manifestation was the decrease in space between the tracheids, which caused the formation of cracks on the macroscopic level. Carbohydrates (cellulose, hemicellulose, and lignin) degraded, which reduced the crystallinity of cellulose, and cracks appeared on the surface of the $S$. psammophila sand barrier. As the aging degree increased, the number of cracks increased, and the cracks continued to extend to both ends. Therefore, the degradation of the S. psammophila sand barrier was mainly caused by shrinkage cracking in the alternated desorptionabsorption aging process, which reduced the ability of the $S$. psammophila sand barrier to resist lodging damage.
\end{abstract}

Keywords: Salix psammophila sand barrier; Structural characterization; Accelerated aging; FTIR spectroscopy; $X$-ray diffraction

Contact information: a: Key Laboratory of State Forest Administration for Desert Ecosystem Protection and Restoration, College of Desert Control Science and Engineering, Inner Mongolia Agricultural University, Hohhot, 010018, China; b: Inner Mongolia Hangjin Desert Ecological Position Research Station, Ordos 017400, P.R. Inner Mongolia China;

${ }^{\dagger}$ The authors contributed equally to this article. *Corresponding author: 13948815709@163.com

\section{INTRODUCTION}

Salix psammophila is a typical desert deciduous shrub or small tree found in grasslands (Gao et al. 2013). It has typical drought resistance characteristics and high temperature resistance, strong resistance to wind erosion, and easy reproduction. $S$. psammophila grows widely in windy and sandy environments with low amounts of rain, and it is mainly distributed in mobile sand dunes, semifixed sand dunes, and lowlands between hills in Northwest China (Gao and Gong 2013). $S$. psammophila sand-barrier is a kind of mechanical sand barrier. It is made of $S$. psammophila wicker, and various configurations of Salix are set on the sand surface to control the direction, speed and structure of wind-sand flow, change the accumulation and wind erosion of the surface (Gao and Gong 2013). In addition, $S$. 
psammophila is an excellent sand barrier material because of its low cost and longlasting and stable protection time. It has been widely used for mechanical sand barriers since the1980s, and it has become one of the key sand control models for the scientific rational and efficient utilization of local resources (Zhang et al.2019). Many studies have shown that the use of fast-growing S. psammophila as a sand barrier remarkable reduces near-surface wind speed and increases surface roughness and vegetation coverage due to its high strength and excellent weather resistance (Xiang-Yu et al. 2008; Gong 2012). In addition, the research shows that the sand barrier improves the microenvironment of sand dunes and accelerates vegetation restoration (Gao et al. 1996).

Because it is a natural biomaterial, when S. psammophila is cut down or cut off, the supply of plant nutrients stops, and the cell tissue dies. The physical and mechanical properties of biomaterials decrease with extended service time, which has adverse effects on their service life (Seldén et al. 2004). Therefore, it is important to evaluate the effects of aging on the performance of a sand barrier. When the sand barrier is exposed to the natural environment for a long time during its service life, the air temperature and humidity constantly change throughout the year and also depend on the time of day. Further, the S. psammophila sand barrier is actually in the state of hygroscopic ad/absorption and desorption. On rainy days, the air humidity is relatively high, and natural biological materials are likely to be in an absorption state. However, when winter winds are constantly blowing, air temperatures drop below $0{ }^{\circ} \mathrm{C}$, and natural biomaterials lose water (Peng et al. $2007)$. Due to the water absorption properties of $S$. psammophila, it is in continual moisture absorption and desorption, and the internal structure and chemical composition of the barrier are irreversibly changed. Cracking, warping deformation, color dim, and strength decrease seriously influence the durability of S. psammophila (Chen et al. 2018). Therefore, it affects the role of wind protection and sand fixation in the field of desertification control. The aging and deterioration of sand barriers caused by changes in environmental humidity should not be underestimated.

S. psammophila is composed of a large number of cells with different shapes, functions, and intersections. The cell cavities and cell walls of the mature, shallow barrier cells have striated holes, and there are gaps between the microcrystals, microfilaments, and filaments in the cell walls (Feng et al. 1996). S. psammophila sand barrier has a certain hygroscopicity that is closely related to its structure, which is mainly due to the hydrophilic groups of cellulose, lignin, and hemicellulose (mainly the hydroxyl and carbonyl groups) in its constituent substances. When water enters the wood's inner cell wall as a gas, it forms hydrogen bonds with the free hydroxyl groups on the cell wall, thereby enabling the material to exchange (hygroscopic ad/absorption and desorption) with the moisture in the atmosphere to achieve an internal moisture balance (Agarwal and Farr 1999; Liu and Zhao 2012). In addition, these molecules are determinants of the physical-mechanical properties of the sand barrier. Therefore, it is essential to study the changes in the main chemical constituents of the sand barrier composed of natural biological material via Fourier transform infrared spectroscopy (FTIR) and X-ray diffraction (XRD) (Traoré et al. 2016). Further, the aging process of $S$. psammophila sand barrier may result in crystallinity changes, which also affect the bending strength, bending elastic modulus, and other valuable physical and mechanical properties (Bhuiyan et al. 2000). 
The research on the moisture and shrinkage cracking of natural biomaterials has been mainly conducted under constant temperature and humidity conditions (static conditions). This method simplifies experimental conditions and theoretical analysis methods and allows for the accumulation of some additional basic data. In fact, due to the constant changes in humidity conditions (dynamic conditions) of the sand barrier, studies under these conditions provide more useful information about practical applications of sand barriers (Kowalski 2001). However, the law of dry shrinkage crack development in the long-term natural environment of S. psammophila sand barrier has not been studied relative to its long-term durability.

In this paper, the phenomenon of drying shrinkage caused by the change of moisture in the environment was investigated. In the field of desertification control, such defects as cracking and warping occur in the process of use. Through artificial simulation, alternated desorption-absorption treatments were performed to accelerate aging of $S$. psammophila sand barrier in the changing moisture of the environment. Through experiments and follow-up analysis, the real-time morphology and mechanical property changes of each group of $S$. psammophila sand barrier after cyclic moisture absorptiondesorption conditions were tracked in real-time. With the help of newly developed observation instruments and characterization methods in the field of materials science, the physical and mechanical properties of S. psammophila sand barrier change due to the change of moisture in the environment was explored. Reasons for the change of the mechanical properties of the damaged S. psammophila sand barrier were analyzed. Thus, the work provides a theoretical basis and literature support for the application of sand barrier pretreatment and the improvement of resource utilization in practice. A further goal is to sustainably improve the ecological environment of northwest China.

\section{EXPERIMENTAL}

\section{Materials}

The test materials were collected in November 2018 in Hangjin Banner, Ordos, Inner Mongolia $\left(108^{\circ} 41^{\prime} 21^{\prime \prime} \mathrm{E}, 40^{\circ} 29^{\prime} 34^{\prime \prime} \mathrm{N}\right)$ according to the National Forestry Industry standard test method for physical and mechanical properties of sand shrubs LY/T 2369 (2014) (Wu et al. 2016). The cuttings of S. psammophila are generally sawed at the end of autumn two years later or at the beginning of the third year. According to a large number of field investigations conducted by the research team in the early stage, the diameters of the branches used for sand barriers were all between $7 \mathrm{~mm}$ and $13 \mathrm{~mm}$. The objective was to reduce the dispersion in the experiment; thus, a total of 20 clusters of representative fresh Salix were randomly selected from the collection area, which were mature in the flat stubble stage and had been growing healthily for 3 years. A branch $20 \mathrm{~cm}$ above the ground with a sawing diameter of $(7 \pm 0.2 \mathrm{~mm}<\mathrm{d}<13 \pm 0.2 \mathrm{~mm})$ was selected as the test material, which was collected and transported to the laboratory. The S. psammophila forest developed under continental monsoon climate, and it has an altitude of $1016 \mathrm{~m}$ and an average annual precipitation volume of $186 \mathrm{~mm}$. There is sufficient sunshine and a large temperature difference between day and night. The annual average temperature is $6.3{ }^{\circ} \mathrm{C}$, the maximum temperature is $38.1^{\circ} \mathrm{C}$, the minimum temperature is $-30.5^{\circ} \mathrm{C}$, and the frostfree period lasts for $135 \mathrm{~d}$.

To minimize the discreteness of test results, the samples with defects from sawing, overly rough surfaces, uneven thickness distributions, nodules, and cracks were removed 
prior to selecting samples. The masses and sizes of the cut specimens were screened. The sample mean length was $70 \mathrm{~mm} \pm 0.2 \mathrm{~mm}$ with an error range of no more than $2 \mathrm{~mm}$.

\section{Experimental design}

Changes in temperature and moisture were simulated by designing an artificial environment. For each alternated desorption-absorption treatment, the S. psammophila sand barrier was immersed in water for $8 \mathrm{~h}$ and then placed in an oven environment at 103 ${ }^{\circ} \mathrm{C} \pm 2{ }^{\circ} \mathrm{C}$ for $6 \mathrm{~h}$. The sand barriers experienced 5 cycles, 10 cycles, 15 cycles, 20 cycles, 25 cycles, 30 cycles, 35 cycles, and 40 cycles. Six samples were randomly selected as a group for each working condition. Then, the samples were placed at a constant temperature of $20{ }^{\circ} \mathrm{C} \pm 2{ }^{\circ} \mathrm{C}$ and a relative humidity of $65 \% \pm 5 \%$. Next, the moisture content was determined which are those national standards mechanics test requirements of approximately $9 \%$ to $15 \%$; and physical and mechanical indicators were tested. After index testing, several samples were selected to be air-dried and ground into wood powder. After the surface dust was removed, they were used to make slices for microscopic observation. The remaining samples in the slices were air-dried and ground into 40-mesh to 60-mesh size powder, which was used to analyze chemical crystal structure and perform spectral and qualitative analysis of chemical groups.

\section{Characterization}

Scanning electron microscopy (SEM) observation

The fabrication of micro-sections was done by sawing from the selected parts with more severe exposure at both ends of $S$. psammophila sand barrier, and these were made into $5 \mathrm{~mm} \times 5 \mathrm{~mm} \times 5 \mathrm{~mm}$ cross and radial slices. First, after 10 d of water bath exhaust, glacial acetic acid was softened at a ratio of 2:1 with a concentration of $30 \%$ hydrogen peroxide for $30 \mathrm{~min}$ (Feng et al. 1996). Then, it was sliced into sections of $30 \mu \mathrm{m}$ in thickness using a sliding microtome (SM 2400; Leika, Beijing, China). The moisture contents were adjusted to below $10 \%$, and transverse sections of wood blocks were polished with a sharp knife, mounted on aluminium stubs with a platinum coating, and then observed by scanning electron microscopy (SEM) (EM-30AX PLUS; COXEM, Daejeon City, Silicon Valley, Korea) at a voltage of $10 \mathrm{kV}$.

\section{FTIR spectroscopy}

Samples collected from different parts of $S$. psammophila sand barrier were used for FTIR analysis. The samples were air-dried for 2 weeks and then powdered. A total of $2 \mathrm{mg}$ and $200 \mathrm{mg}$ potassium bromide samples were mixed evenly by the $\mathrm{KBr}$ method and placed in the mold. The circular thin sample was made under the conditions of 8 to $10 \mathrm{MPa}$ and $2 \mathrm{~min}$. A FTIR spectrometer (TENSON 27; Bruker Corp., Beijing, China) was used with $4 \mathrm{~cm}^{-1}$ resolution in the $4000 \mathrm{~cm}^{-1}$ to 400 $\mathrm{cm}^{-1}$ absorbance range. Each sample were collected 32 scans, and the spectral data were calibrated and normalized at baseline.

\section{$X$-ray diffraction}

The S. psammophila sand barrier sample was treated using an X-ray diffractometer (Philips X-Pert; Panalytical, Almelo, Netherlands) using $\mathrm{Cu} \mathrm{K \alpha}$ radiation $(k=0.154 \mathrm{~nm})$. The working conditions were as follows: $40 \mathrm{kV}$ and 30 $\mathrm{mA}, 2 \mathrm{~s} / \mathrm{step}, 0.02 \%$ step. The sample was spread over a range of $2 \theta=5^{\circ}$ to $40^{\circ}$ 
(Bhuiyan et al. 2001; Zhang et al. 2011). The degree of crystallinity (CrI) was determined via the Segal method (Li 2003) Eq. 1,

$$
\operatorname{CrI}(\%)=\left(I_{002}-I_{\mathrm{am}} / I_{002}\right) \times 100
$$

where $\mathrm{CrI}(\%)$ is the crystallinity degree, $I_{002}$ is the diffraction intensity of the crystallizing area from the (002) plane at $2 \theta=22.5^{\circ}, I_{\mathrm{am}}$ is the intensity of the amorphous background scatter measured at $2 \theta=18^{\circ}, I_{002}$ represents both crystalline and amorphous material, and $I_{\mathrm{am}}$ represents only the amorphous material (Inagaki et al. 2010).

\section{Determination of physical-mechanical properties}

The samples of $S$. psammophila sand barrier segments from each sampling point were placed in a constant temperature and humidity box with a temperature of $(20 \pm 2){ }^{\circ} \mathrm{C}$ and a relative humidity of $(65 \pm 5) \%$. The moisture content was adjusted to reach $9 \%$ to $15 \%$, as specified by the national standard mechanical test requirements, and physical and mechanical tests were conducted. The specimens were weighed with an electronic balance (accuracy of $0.001 \mathrm{~g}$ ). The radial, chord, and longitudinal thickness dimensions of the specimens were measured using an electronic digital caliper, and the average value was determined based on tests at 5 points. At the same time, the position was measured and identified. The mass was measured by an electronic balance, accurate to $0.002 \mathrm{~g}$, and detailed records were made. The diameter and length of the samples were measured with an electronic digital display caliper, and the physical characteristics of the samples were measured. The physical properties of the $S$. psammophila sand barrier samples were determined according to LY/T 2369 (2014). The basic density $\left(D, \mathrm{~g} / \mathrm{cm}^{-3}\right)$ was determined based on the measured oven-dry weight and green volume. Drying shrinkage $(S, \%)$ was estimated with air- and oven-dried samples. The volume of the sample was determined using a water displacement method. The mechanical properties, such as modulus of rupture (MOR, MPa) and modulus of elasticity (MOE, GPa), of the cross-sections were determined according to LY/T 2369 (2014) using a universal testing machine (model TY-8000; Tianyuan, Jiangsu, China).

\section{RESULTS AND DISCUSSION}

\section{Performance of Salix psammophila Sand Barrier}

After 5 to 40 alternated desorption-absorption cycles, the S. psammophila sand barrier had different degrees of cracking. The cracks developed from the medullary center in the radial direction and extended to the adjacent side until fracture occurred. The discreteness of the $S$. psammophila sand barrier materials was larger. Additionally, test operation inevitably contributed to test error, such as each sample in the oven position and the position in the water tank resulted in a slightly different external humidity environment, etc., the same aging degree of samples show different crack development. After 10 cycles, local microcracks were generated (Fig. 1a).

After 20 cycles, the number of cracks increased, and the length of the cracks increased as the number of alternated desorption-absorption cycles increased (Fig. 1b). After 30 cycles, the cracks of some specimens extended from the side and ran through the entire adjacent longitudinal plane (Fig. 1c). Serious sample failure was observed after 40 
cycles, as there were multiple through-penetration fractures (Fig. 1d). After 10 cycles, small cracks appeared on the side of the sample (Fig. 1e), and the cracks became longer and more numerous as the number of cycles increased. However, these cracks were not present in the adjacent surface. After 20 cycles, the cracks in the longitudinal plane became discontinuous (Fig. 1f). Following 30 cycles, the $S$. psammophila had very few cracks on the side of the fracture propagation and throughout the adjacent vertical planes, but they did not result in a sample fracture (Fig. 1g and Fig. 1h), which may have been due to the poor individual sample quality.
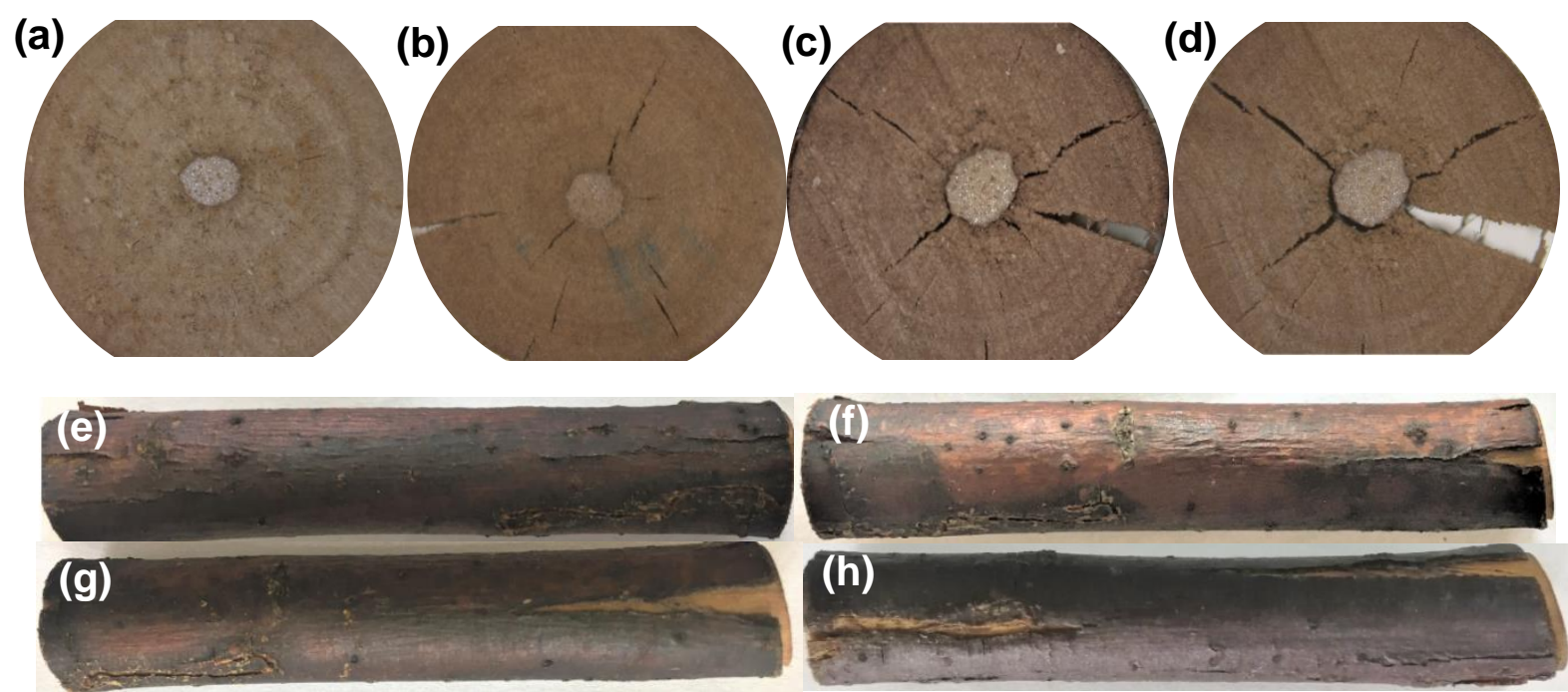

Fig. 1. Radial and cross-section morphological changes of aging (a and e: 10 cycles, b and f: 20 cycles, $c$ and $g: 30$ cycles, $d$ and $\mathrm{h}: 40$ cycles)

\section{Physical and mechanical properties}

According to Figs. 2a, 2b, and 2c, the S. psammophila sand barrier experienced dry shrinkage after continuous desorption-absorption alternated aging. The radial, chord, and portrait shrinkage rates of the $S$. psammophila sand barrier with the increased number of alternated desorption-absorption cycles and chord contraction was obvious. 
(a)

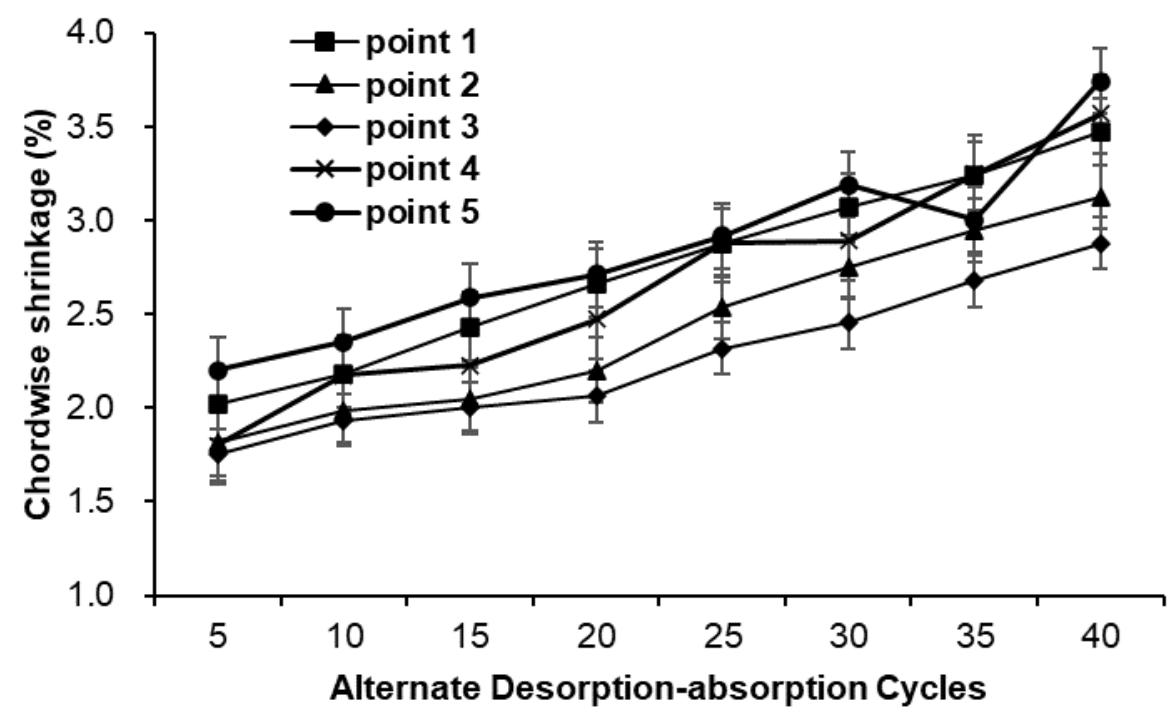

(b)

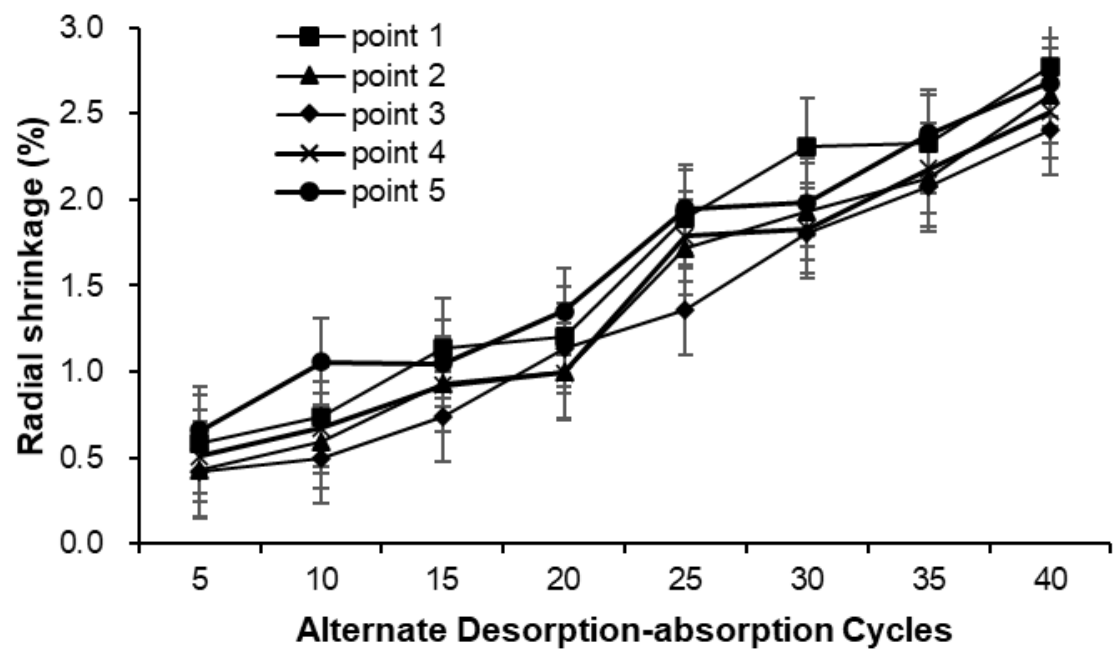

(c)

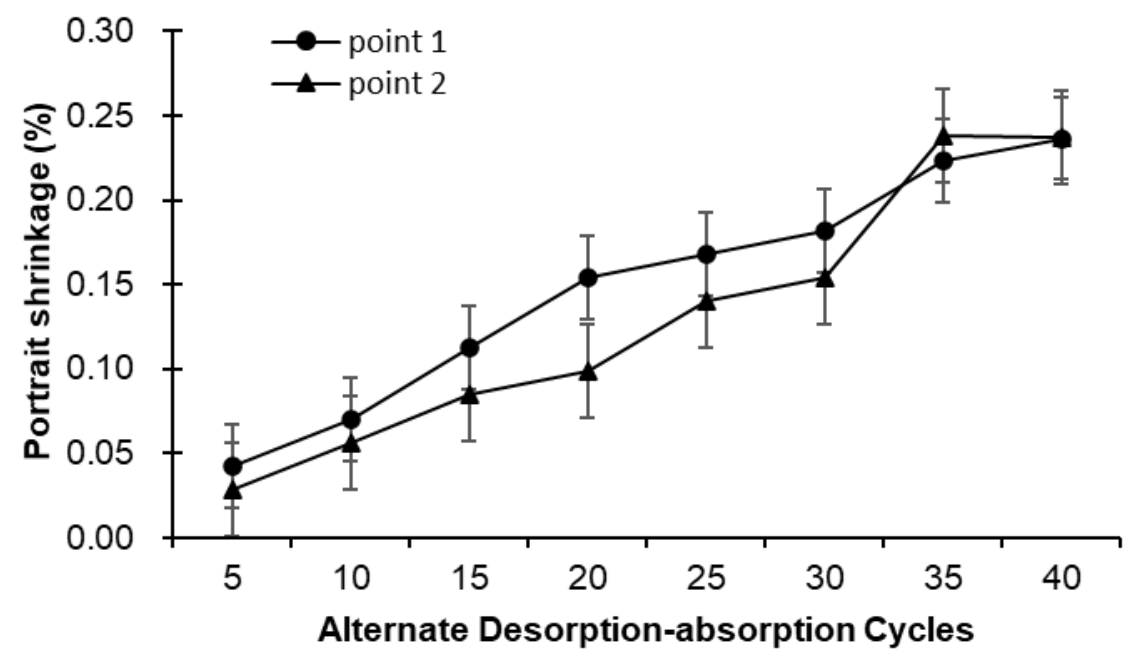



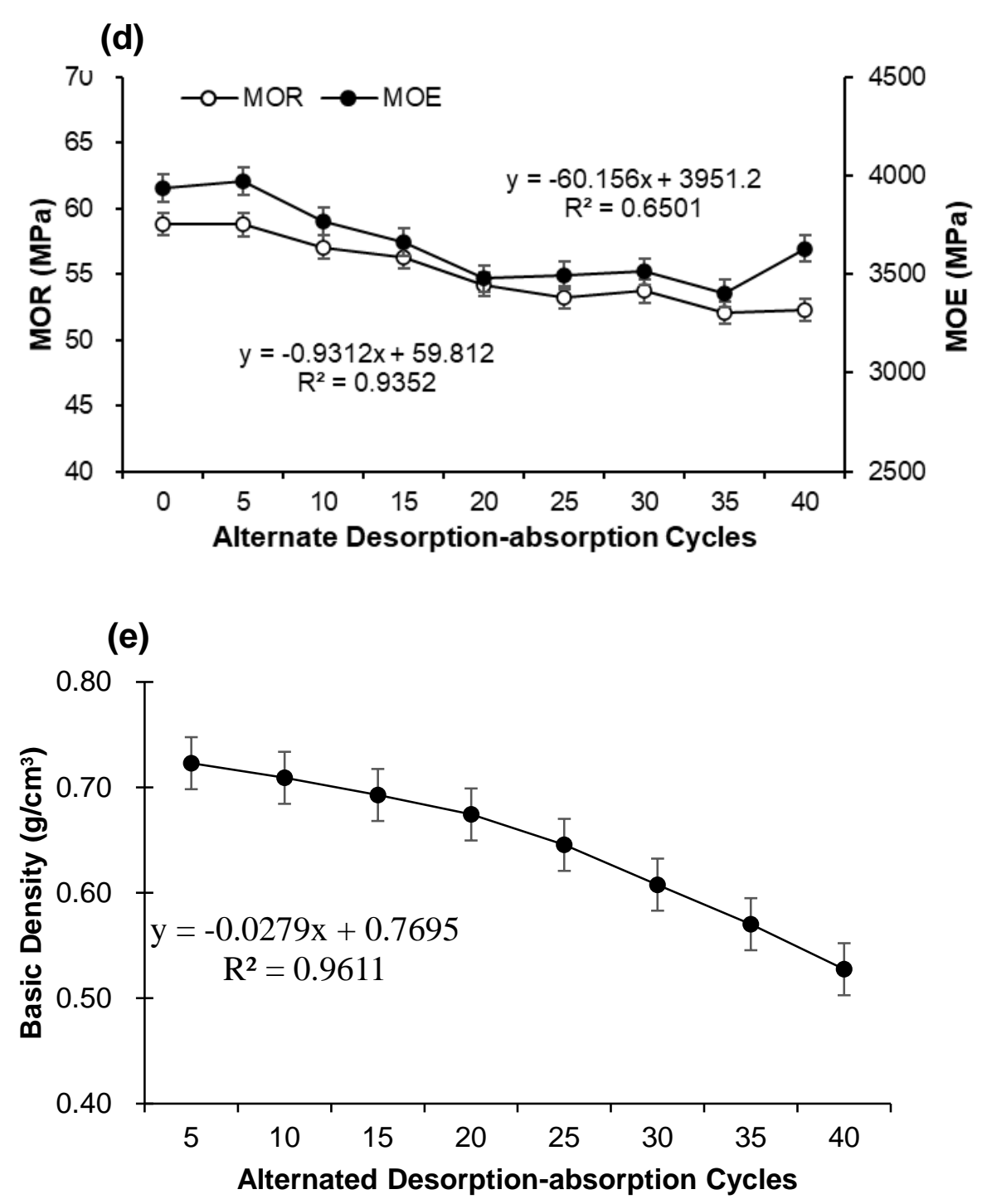

Fig. 2. Physical-mechanical properties (a: Chordwise shrinkage, b: Radial shrinkage, c: Portrait shrinkage, d: MOR and MOE, e: Basic density,); Error bars represent standard errors.

The extent of dimensional change observed was longitudinal $>$ radial $>$ transverse. According to the dry shrinkage rate of the five measured points of the $S$. psammophila sand barrier, the deformation in the middle of $S$. psammophila sand barrier was smaller with alternated desorption-absorption cycles, and the deformation was more obvious as it developed towards both ends. This suggests that, in the process of deformation, the intermediate size changed the least, and the remaining two sections changed the most. After the alternation cycles, the drying shrinkage rate of $S$. psammophila sand barrier string changed. This occurred because, after being cut down and truncated, the catheter at both ends was exposed, which allowed end cracks to form easily. After alternated desorptionabsorption cycles, the surface cracking of $S$. psammophila sand barrier gradually led to shrinkage. However, after alternated absorption-desorption cycling, the content and hydrophilicity of the polar groups were changed (Li et al. 2007; Peng et al. 2007). Density is an important physical property of natural biomaterials that affects the drying shrinkage 
of wood (Lin 1997). After alternated absorption and desorption, polar functional groups, such as $\mathrm{C}=\mathrm{O}, \mathrm{C}-\mathrm{O}$ and $\mathrm{O}-\mathrm{H}$, were reduced, and the hydrophilicity of the S. psammophila sand barrier was weakened, which resulted in a certain change in density during hygroscopicity or desorption. With the increase of the number of alternated absorptiondesorption cycles, the absolute dry density of $S$. psammophila sand barrier showed a slow and gradual decline trend, and the relationship between the two presented a linear function change, and $R^{2}=0.9611$. Analysis of variance showed that the changes of different cycles of alternated absorption-desorption were significantly different $(\mathrm{P}<0.05)$, and the rate of decrease of density decreased $(14.7 \%)$.

The mechanical properties of natural biomaterials are directly related to their density and physical properties of dry shrinkage. Modulus of rupture (MOR) and modulus of elasticity (MOE) of natural biomaterials are the most important mechanical properties in the utilization of biological resources (Kol et al. 2010; Clair and Thibaut 2014). It is shown in Fig. 2d, the mechanical properties of MOR and MOE decreased to a certain extent after 0 to 40 cycles of alternated desorption-absorption, both of which decreased significantly after the 20 to 30 cycles of alternated desorption-absorption. The relationship between the two was a linear function, and MOR and MOE were $R^{2}=0.9352$ and $R^{2}=0.6501$, respectively. Analysis of variance showed that MOR and MOE with different cycles of alternated desorption-absorption were significantly different $(\mathrm{P}<0.05)$. The MOR decrease for S. psammophila sand barrier was $12.5 \%$, which was consistent with the changes in density and shrinkage. In contrast with MOR, MOE changes lacked regularity, which was consistent with the literature. However, the overall trend was downward, as an $8.4 \%$ decrease in MOE was observed. The decrease was slow in the early stage of aging, but it became faster as the number of cycles increased, and it ultimately stabilized. After 10 cycles, cracks on the $S$. psammophila sand barrier may have begun to form, and the aggravation of aging caused the cracks to extend and develop. In addition, the number of cracks continued to increase, and they extended to both ends. The main cause of strength loss was the degradation of cellulose and hemicellulose (Hillis and Rozsa 1985). This showed that the alternated damage of desorption-absorption had a notable effect on MOR.

The results of physical and technical property testing of the S. psammophila sand barrier after alternated desorption-absorption indicated that the drying shrinkage rate of the sand barrier with increased number of alternated times and the chord drying shrinkage rate was greater than the longitudinal drying shrinkage rate. Cracks were found to increase and extend to both ends. The mechanical properties of the sand barrier also decreased.

\section{Microstructural analysis}

The materials in each test period were sectioned for observation. Figures $3 \mathrm{a}$ and $3 \mathrm{~b}$ show the microstructure of untreated materials and the $500 \mathrm{X}$ cross-section magnified sample after 40 cycles of alternated desorption-absorption. As the number of moisture cycles increased, the overall internal tracheid spacing decreased, and the transverse surface became rough. Due to the increase in brittle deformation, the cellular structure collapsed, cell walls contracted inward, and the ray structure was affected. This influenced the macroscopic performance for the number and width of cracks increase increased. With the increase of alternated desorption-absorption cycles, the permeability of $S$. psammophila sand barrier became decreased, which was related to the structure of S. psammophila. 


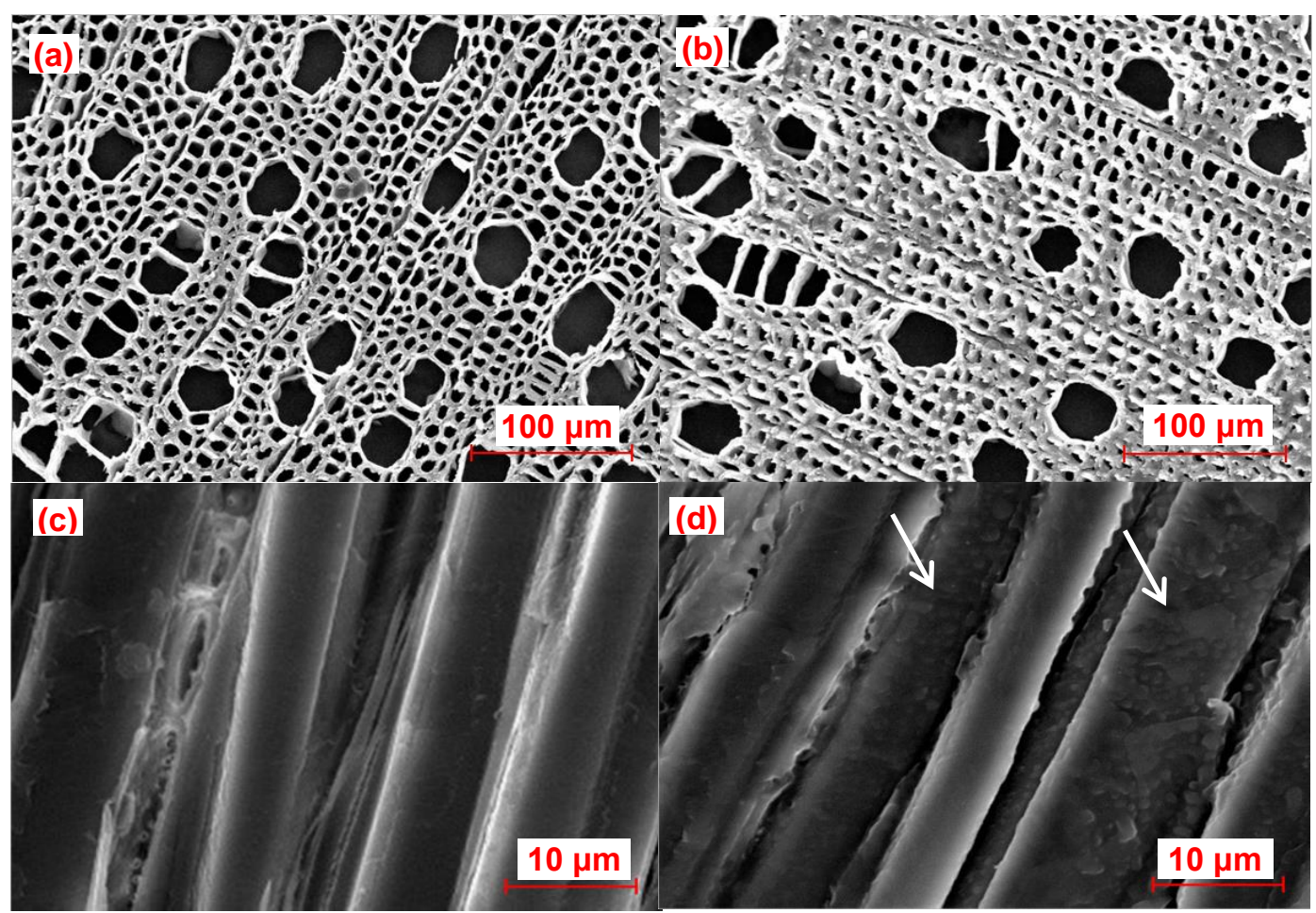

Fig. 3. SEM photographs (a: 0 cycles cross-section, b: 40 cycles cross-section, c: 0 cycles radial -section, $\mathrm{d}$ : 40 cycles radial -sections.); scale bar is 100 and $10 \mu \mathrm{m}$

Figures $3 \mathrm{c}$ and $3 \mathrm{~d}$ show the SEM photographs with an $1000 \mathrm{X}$ radial-section of $S$. psammophila sand barrier. Note the rare precipitate in the wood ray thin wall cells, which clogs the cell wall. The natural biological material extract is deposited in the conduit or cell wall, which affects the permeability by blocking the channel where the fluid penetrates, making it act as a structural barrier and making it difficult for water to move through the $S$. psammophila sand barrier. This result is consistent with the results of Jiang et al. (2003), who found that the changes of extracted species would cause changes in the properties of the internal and external surfaces of natural biomaterials, change their interface properties, and affect the diffusion and flow of fluids (water) in natural biomaterials (Jiang et al. 2003; Li et al. 2007). At the same time, in the degradation process of natural biological materials, fats, waxes, and other extracts have migrated to the surface, and degradation products of polysaccharides such as hemicellulose and cellulose amorphous region can still form new extractable substances (Cheng et al. 2016). Therefore, due to the poor permeability and increased shrinkage rate in the process of alternated desorption-absorption, shrinkage occurred easily in the drying process. This occurred due to water loss and changes in internal anatomical structure.

Through observation and analysis of the microscopic structure, the law of deterioration and deformation of $S$. psammophila sand barrier was summarized. In a continuous alternated desorption-absorption cycle, water molecules pass through the perforations in the wall of the catheter and form the parenchyma cells. At the same time, the change of the extracted material blocked the pores, and the water movement was difficult, which eventually led the cell wall to undergo dry shrinkage and deformation. As a result, the cell wall and even the entire S. psammophila sand barrier were changed in size and shrank in volume.

Wang et al. (2020). "Accel. aging Salix psammophila," BioResources 15(3), 6696-6713. 6705 


\section{FTIR analysis}

FTIR spectroscopy was used to determine the strength and functional groups of cellulose, hemicellulose, and lignin bands in the samples. These bands show the structural changes of biological materials during the aging process, which lead to degradation or modification (Pandey 1999; Traoré et al. 2016). The chemical structure of cellulose, hemicellulose, and lignin, and the chemical functional groups and chemical bonds of $S$. psammophila sand barrier were changed when the sand barrier aged due to environmental factors.

Fourier transform infrared spectroscopy was used to analyze the alternated desorption-absorption of $S$. psammophila sand barrier. To facilitate comparison, the original infrared spectra of the collected samples were normalized at the highest point $\left(3400 \mathrm{~cm}^{-1}\right)$. Figure 4 shows the FTIR results for samples with 0 cycles (untreated) 10 cycles, 20 cycles, 30 cycles, and 40 cycles of desorption-absorption after normalization. The two peaks near $3400 \mathrm{~cm}^{-1}$ and $2900 \mathrm{~cm}^{-1}$ are considered the most important features of natural biological materials in the infrared spectrum, as they represent the absorption peaks of O-H and C-H stretching vibrations, respectively (Müller et al. 2003).

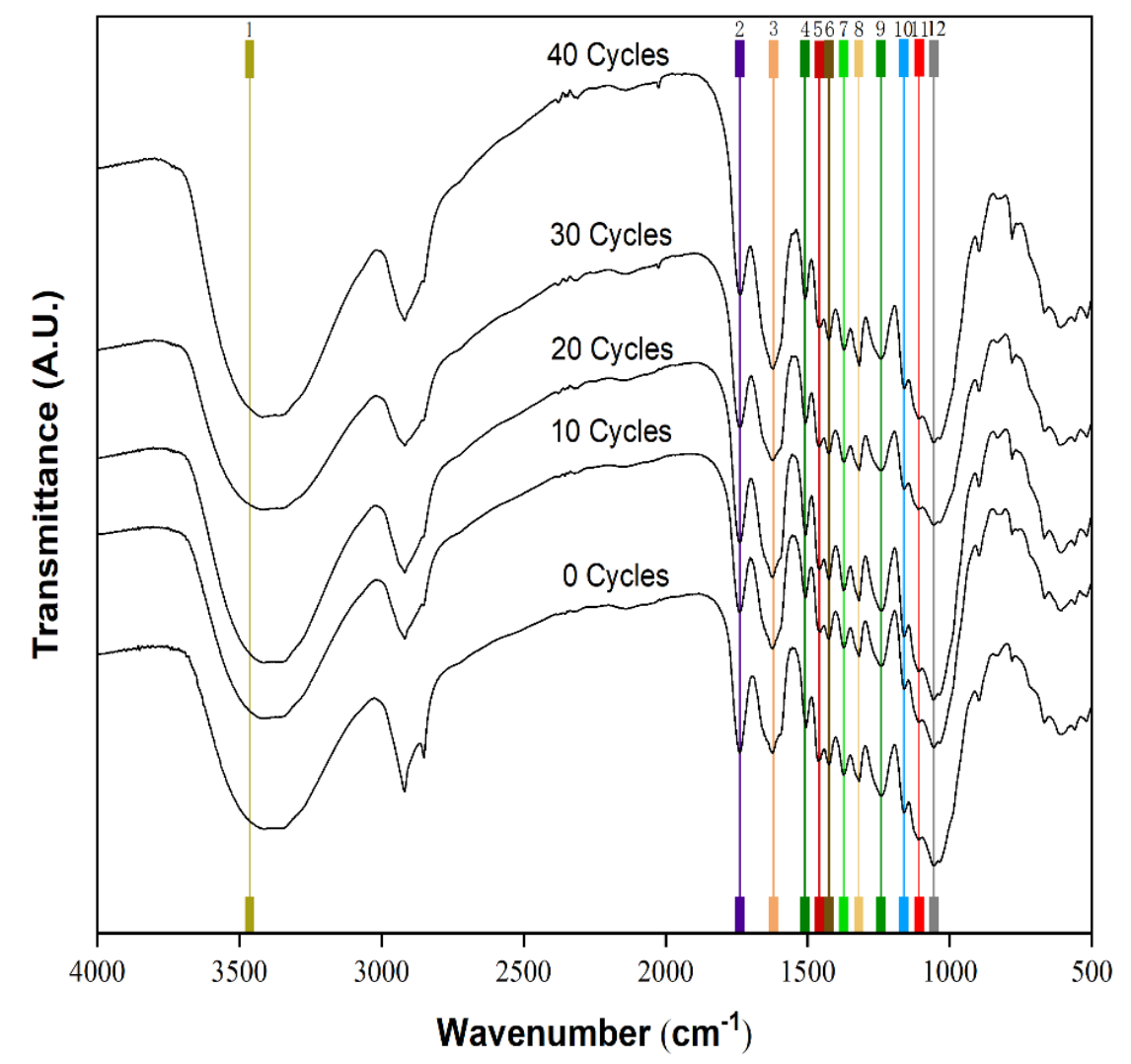

Fig. 4. Infrared spectra and their derivatives in the $4000 \mathrm{~cm}^{-1}$ to $400 \mathrm{~cm}^{-1}$ region. Twelve different peaks are highlighted in different colors and wavelength assignment of the following peak numbers showed in Table 1. 
Table 1. FTIR Spectrum Wavelength Assignment of Untreated and Alternated Desorption-absorption Cycle of S. psammophila Sand Barrier

\begin{tabular}{|c|l|c|}
\hline Peak No. & \multicolumn{1}{|c|}{ Wavelength Assignment } & $W\left(\mathrm{~cm}^{-1}\right)$ \\
\hline 1 & $\mathrm{O}_{2}-\mathrm{H}_{2} \ldots \mathrm{O}_{6}$ intramolecular stretching modes in cellulose & 3416 \\
\hline 2 & Unconjugated C=O in organic molecules (hemicellulose) & 1740 \\
\hline 3 & $\mathrm{C}=\mathrm{O}$ stretching in conjugated double bond & 1624 \\
\hline 4 & Aromatic skeletal ring in lignin & 1506 \\
\hline 5 & $\mathrm{C}-\mathrm{H}$ deformation of lignin and carbohydrates & 1464 \\
\hline 6 & $\mathrm{C}-\mathrm{H}$ deformation in organic molecules in general & 1425 \\
\hline 7 & $\mathrm{C}-\mathrm{H}$ deformation in cellulose and hemicellulose & 1373 \\
\hline 8 & $\mathrm{C} 1-\mathrm{O}$ vibrations in syringyl units and C-H vibrations in cellulose & 1319 \\
\hline 9 & C-O stretch in lignin and C-O linkage in guaiacyl aromatic groups & 1242 \\
\hline 10 & C-O-C vibrations in cellulose and hemicellulose & 1161 \\
\hline 11 & Aromatic skeletal and C-O stretch in lignin & 1109 \\
\hline 12 & C-O stretch in cellulose and hemicellulose & 1057 \\
\hline
\end{tabular}

Compared with untreated sand barrier, the absorption peak of the stretching vibrating hydroxyl group at $3415 \mathrm{~cm}^{-1}$ moved to a higher position and its absorbance decreased after alternated desorption-absorption, which indicates that alternated desorption-absorption reduced the association degree of the hydroxyl groups. The absorption intensity at $2900 \mathrm{~cm}^{-1}$ was remarkable reduced, which indicates that the $S$. psammophila sand barrier exhibited aging degradation as the number of cycles increased. For natural biological materials, the changes of various groups in their chemical components are reflected in the fingerprint region of the infrared spectrum $\left(800 \mathrm{~cm}^{-1}\right.$ to $1,800 \mathrm{~cm}^{-1}$ ). At $1740 \mathrm{~cm}^{-1}, 1620 \mathrm{~cm}^{-1}, 1510 \mathrm{~cm}^{-1}, 1460 \mathrm{~cm}^{-1}$, and $1425 \mathrm{~cm}^{-1}$, obvious absorption peaks appeared in the S. psammophila sand barrier, and they changed with increased aging time. The absorption peak at $1739 \mathrm{~cm}^{-1}$ was attributed to the tensile vibration of $\mathrm{C}=\mathrm{O}$ in the acetyl group, and the intensity weakening was attributed to the carboxyl group of hemicelluloses (Teacă et al. 2013). After 40 cycles, the characteristic peak at $1739 \mathrm{~cm}^{-1}$ fluctuated noticeably, and the characteristic peak at $1620 \mathrm{~cm}^{-1}$ expanded considerably. These results indicate that the carboxyl group in hemicellulose was decomposed, which affected the characteristic peaks at $1242 \mathrm{~cm}^{-1}, 1161 \mathrm{~cm}^{-1}$, and 1109 $\mathrm{cm}^{-1}$ with a small range of fluctuations (Takei et al. 1997), and the absorption peak at 1510 $\mathrm{cm}^{-1}$ expands and contracts noticeably. At $1510 \mathrm{~cm}^{-1}$, the aromatic benzene ring skeleton attributable to lignin vibrates, and the absorption peak had undergone significant expansion and contraction, indicating that lignin had undergone a greater degree of degradation. The vibrations of the benzene ring skeleton at $1506 \mathrm{~cm}^{-1}$ and $1464 \mathrm{~cm}^{-1}$ decreased the absorption intensity, which illustrates that the lignin had a small change in the lignin phenol ether bond $\mathrm{C}-\mathrm{O}-\mathrm{C}$. The wave positions of C-O-C, ester group, saturated fat ether, and alcohol C-O on the lignin phenol ether bond and the piaran sugar ring were for the most part unchanged, but the absorption intensity was weakened. This was because after the alternations, the ethers, alcohols, esters, lignans, and low molecular weight sugars of the extracts of $S$. psammophila sand barrier were changed. Figure 3 shows that the deformation and vibration of $1373 \mathrm{~cm}^{-1}$ methyl C-H of cellulose and hemicellulose, the characteristic absorption peaks of lignin $\left(1506 \mathrm{~cm}^{-1}\right.$ and $\left.1242 \mathrm{~cm}^{-1}\right)$ were weakened, and the characteristic absorption peaks of cellulose and hemicellulose were also weakened $\left(1161 \mathrm{~cm}^{-1}\right.$ and $\left.1109 \mathrm{~cm}^{-1}\right)$. The intensity of the vibration absorption peak of the aromatic ring carbon skeleton characterized by O-H association absorption band at $1109 \mathrm{~cm}^{-1}$ and $1506 \mathrm{~cm}^{-1}$ decreased 
slightly after 20 cycles. This was mainly due to the degradation of lignin and hemicellulose at $103{ }^{\circ} \mathrm{C}$ to $105^{\circ} \mathrm{C}$ after alternated drying.

The changes of functional groups in natural biomaterials are indicated by the positions of spectral peaks and the relative absorption intensity of absorption peaks in the infrared spectra. The infrared spectra are consistent with the presence of the hydroxyl group, methyl group, methylene group, carbonyl group, carboxyl group, benzene ring, carbon-chlorine bond, ether bond, nitrogroup, C-O single bonds, and triple bonds or cumulative double bonds. Compared with untreated sand barrier, the hydroxyl absorbance of $S$. psammophila sand barrier decreased after hygroscopic desorption. The $\mathrm{C}=\mathrm{O}$ in the non-conjugated aldehydes, ketones, carbonyl groups, acyl groups, or esters weakened. $\mathrm{C}=\mathrm{O}$ peaks appear in conjugated aldehydes, ketones, carbonyl groups, acyl groups, or esters. The $\mathrm{C}-\mathrm{O}$ absorption intensity of the phenolic ether bond $\mathrm{C}-\mathrm{O}-\mathrm{C}$ on the pyranose ring, ester group, saturated fat ether, and alcohol decreased, and the absorption intensity of the benzene ring varied. Therefore, the contents and hydrophilicity of the polar functional groups of S. psammophila sand barrier changed during the alternated desorption-absorption cycling. The infrared spectrogram of sand barrier before and after alternated desorptionabsorption showed that the hydrophilic group of the sand barrier decreased as the number of drying cycles increased. Therefore, the redistribution and chemical reaction of the extract S. psammophila sand barrier chinensis reduced its hydrophilicity and poor permeability. As a result, the dry shrinkage rate of $S$. psammophila sand barrier rose with the alternated desorption-absorption cycles.

\section{X-ray Diffraction Analysis}

Where cellulose macromolecular chains are most dense, the chains are regularly arranged in a parallel and well-directed manner that resembles the characteristics of some crystals; thus, they are called crystalline regions (Love et al. 2020).

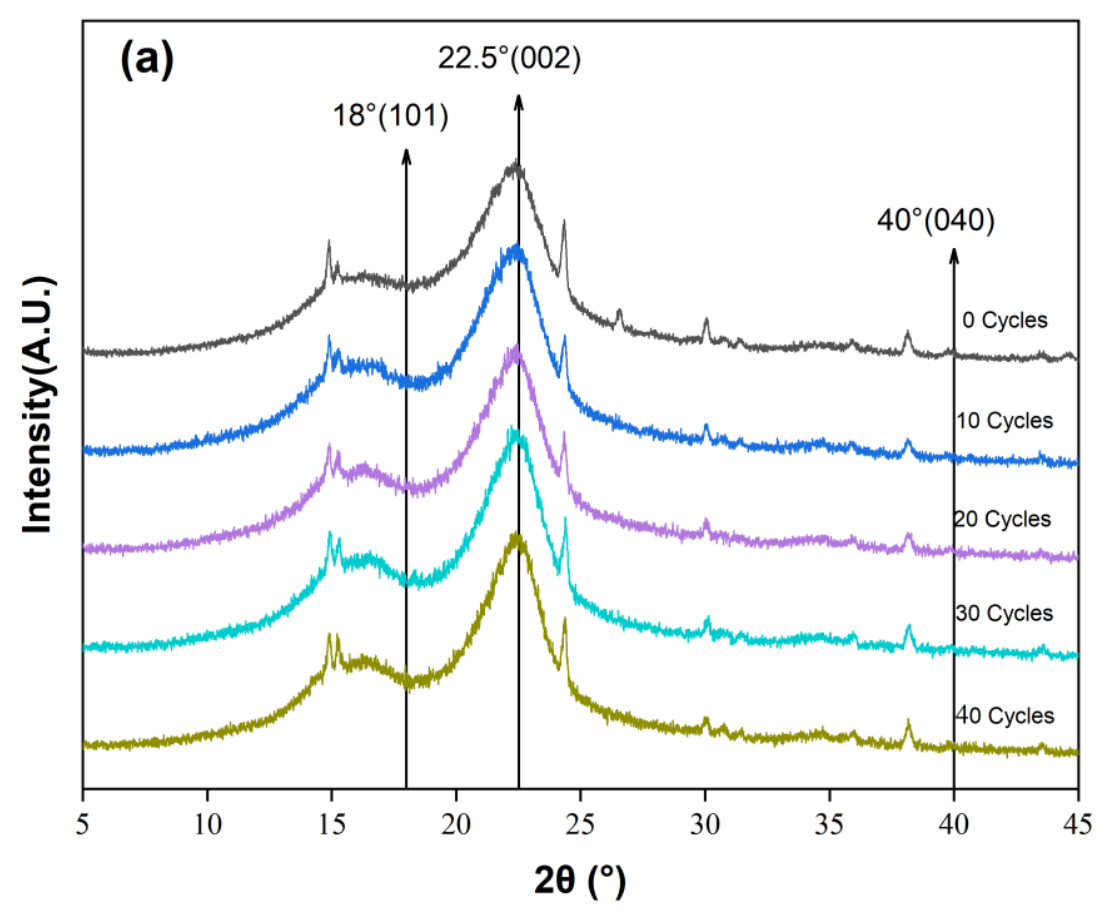




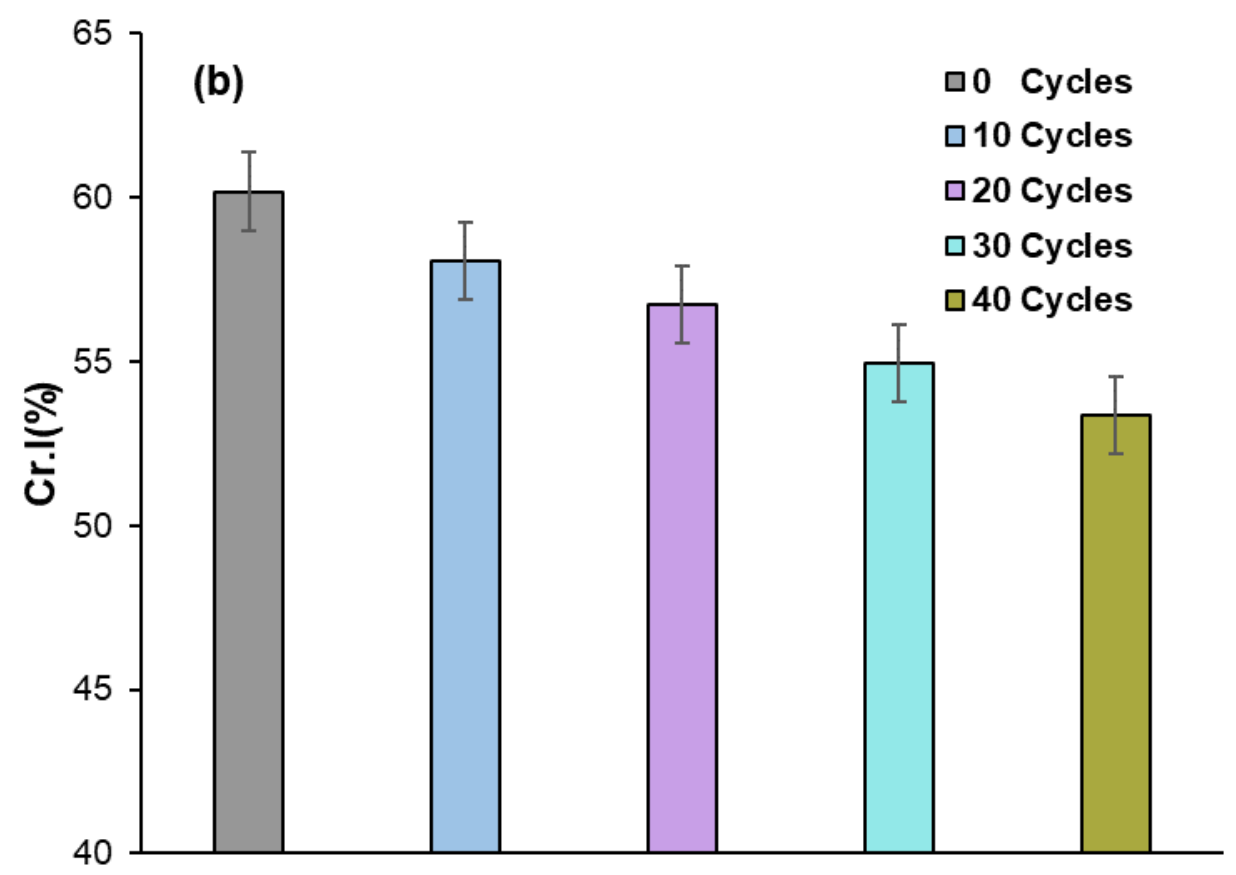

Alternate Desorption-absorption Cycles

Fig. 5. XRD diffractogram (a: Alternated desorption-absorption of sand barrier, b: Vertical position); error bars represent standard errors.

In the main components of $S$. psammophila, cellulose has a certain degree of crystallinity, which refers to the percentage of cellulose crystallization area in the whole cellulose. Crystallinity reflects the physical and chemical properties of natural biomaterial fibers (Xia et al. 2018). As cellulose crystallinity increases, the flexural strength, flexural elastic modulus, and other mechanical properties of natural biomaterials generally increase. For instance, the material brittleness increases, the toughness decreases, and reactivity decreases. For aged materials, the decrease in mechanical strength caused by degradation of cellulose is accompanied by a decrease in crystallinity. Therefore, X-ray diffraction analysis could help to characterize the aging process of the cellulose crystal area of salicorus sand barrier.

Figure 5 shows the XRD pattern and relative crystallinity of the $S$. psammophila sand barrier after hygroscopicity and desorption alternation for 0 cycles, 10 cycles, 20 cycles, 30 cycles, and 40 cycles. Figure 4 a shows the crystal diffraction peaks at the X-ray diffraction angle $(2 \theta)$ near $18^{\circ}, 22.5^{\circ}$, and $35^{\circ}$, which represent the crystal diffraction intensity of the cellulose (101, 002, and 040) planes of $S$. psammophila sand barrier, respectively. Among them, $2 \theta=22.5^{\circ}$ reflects the crystal diffraction peak of cellulose (002). The positions of cellulose diffraction peaks were consistent, which indicated that the crystalline structure of $S$. psammophila sand barrier did not change.

The absorption intensity of the cellulose crystal diffraction peak decreased as the number of desorption-absorption alternated increased, which indicates that the relative crystallization content of cellulose crystal region decreases. With the degradation of lignin, under the long-term influence of environmental moisture, the partial crystallization area of cellulose changes to an amorphous state, leading to the decrease of relative crystallinity. Figure $5 \mathrm{~b}$ shows the wave valley near $2 \theta=18^{\circ}$, which is the scattering intensity of the 
amorphous diffraction fiber of S. psammophila sand barrier. After 40 cycles of alternated desorption-absorption, the diffraction intensity in the amorphous region of the salsa sand barrier was lower, indicating that the amorphous region was degraded during the transformation of the cellulose crystal region to the amorphous region, leading to a decrease in the diffraction intensity of the amorphous region and a decrease in its relative crystallinity. In addition, the relative crystallinity of wood was calculated by using the peaking area method. After 40 cycles of moisture absorption and desorption, the cellulose crystallinity decreased $6.8 \%$, and the FTIR analysis results showed that serious degradation of cellulose and fiber occurred. This degradation was because in the high temperature range of $103{ }^{\circ} \mathrm{C}$ to $105{ }^{\circ} \mathrm{C}$, hemicellulose degrades with the removal of the acetyl group to produce acetic acid, which destroys the structure of cellulose and reduces the crystallinity of cellulose. The physical-mechanical properties of $S$. psammophila sand barrier exposed to an alternated desorption-absorption aging process were analyzed. During the aging process, the base density decreased and shrank due to decreases in cellulose and hemicellulose content. In addition, the variation in MOR was consistent with that in cellulose crystallinity (González-Peña et al. 2009). The crystallinity of cellulose was also found to affect the mechanical properties (Bhuiyan et al. 2000).

According to the results of relative crystallinity analysis, the absorption intensity of the cellulose crystal diffraction peak decreased as the number of desorption-absorption alternated increased, which indicates that the relative crystallization content of cellulose crystal region decreased. The degradation of cellulose and hemicellulose may be due to the removal of acetyl groups from hemicellulose and the formation of acetic acid, which destroys the structure of neovulin and reduces the crystallinity of cellulose.

During the process of using the S. psammophila sand barrier, the change of moisture content leads to the change of its dimensional stability and apparent cracking deformation. After 0 to 40 cycles, the mechanical properties of $S$. psammophila sand barrier were decreased. Based on the analysis of microstructures, chemical bonds and crystallinity of cellulose, it can be easily understood that the reduction of mechanical properties in the process of alternated desorption-absorption degradation is positively related to the loss of carbohydrate and other chemical components. These results indicate that the change of moisture content is a major factor in the deterioration of $S$. psammophila sand barrier.

\section{CONCLUSIONS}

1. The continuous hygroscopicity and desorption of S. psammophila sand barrier weakened the physical-mechanical properties of wood by alternated accelerated aging treatment. The decrease was slow in the early stages of aging, but it became faster and stabilized as the number of alternated desorption-absorption cycles increased, which was related to changes in anatomical characteristics and chemical composition.

2. At the micro level, the S. psammophila sand barrier was characterized by a decrease in the space between the tracheids, which caused the formation of cracks at the macro level.

3. During the aging process, carbohydrates (cellulose, hemicellulose, and lignin) degraded, which reduced the crystallinity of cellulose. 
4. As the number of alternated desorption-absorption cycles increased, the number of cracks in S. psammophila sand barrier increased. The shrinkage crack of the surface caused by the change of moisture in the environment were the main reason for the decline in physical and mechanical properties.

5. The depolymerization of carbohydrate and other chemical components and reduction of crystals help to reduce the physical and mechanical properties of wood samples. Therefore, the degradation of sand barrier is mainly caused by shrinkage cracking in the process of changing moisture content in the environment.

\section{ACKNOWLEDGMENTS}

The research was supported by the National Natural Science Foundation of China (41861044), "The enhancement mechanism of atmospheric - sand dynamic process on Salix sand barrier resistance."

\section{REFERENCES CITED}

Agarwal, N., and Farris, R. J. (1999). "Water absorption by acrylic-based latex blend films and its effect on their properties," Journal of Applied Polymer Science 72(11), 1407-1419. DOI: 10.1016/j.soilbio.2013.03.027

Bhuiyan, M. T. R., Hirai, N., and Sobue, N. (2000). "Changes of crystallinity in wood cellulose by heat treatment under dried and moist conditions," Journal of Wood Science 46(6), 431-436. DOI: 10.1007/BF00765800

Chen, K., Qiu, H. X., and Zhu, Z. M. (2018). "Bearing capacity of timber beams with shrinkage cracks," Journal of Civil, Architectural and Environmental Engineering 40(1), 39-47. DOI: 10.11835/j.issn.1674-4764.2018.01.006

Cheng, S., Huang, A., Wang, S., and Zhang, Q. (2016). "Effect of different heat treatment temperatures on the chemical composition and structure of Chinese fir wood," BioResources 11(2), 4006-4016. DOI: 10.15376/biores.11.2.4006-4016

Clair, B., and Thibaut, B. (2014). "Physical and mechanical properties of reaction wood," in: The Biology of Reaction Wood, Springer, Berlin, Germany, pp. 171-200.

Feng, L. Q., Gao, X. X., and Wang, X. M. (1996). "Microstructure and chemical composition analysis of Salix psammophila," Journal of Inner Mongolia Forestry University 18(1), 38-42.

Gao, Y., and Yi, Y. (1996). "Studies on suitable vegetational cover rate for Salix psammophila forest," Inner Mongolia Forestry Science \& Technology 3(Z1), 38-42.

Gao, Y., and Gong, P. (2013). Salix Sand Barrier, Beijing Science Press, Beijing, China.

Gong, P. (2012). The Corrosion Processes of Salix Sand-barrier and the Effect of Anticorrosion, Ph.D. Dissertation, Inner Mongolia Agricultural University, Hohhot, Inner Mongolia.

González-Peña, M. M., Curling, S. F., and Hale, M. D. C. (2009). "On the effect of heat on the chemical composition and dimensions of thermally-modified wood," Polymer Degradation and Stability 94(12), 2184-2193. DOI: 10.1016/j.polymdegradstab.2009.09.003 
Hillis, W. E., and Rozsa, A. N. (1985). "High temperature and chemical effects on wood stability. Part 2. The effect of heat on the softening of radiata pine," Wood Science and Technology 19(1), 57-66. DOI: 10.1007/BF00354753

Inagaki, T., Siesler, H. W., Mitsui, K., and Tsuchikawa, S. (2010). "Difference of the crystal structure of cellulose in wood after hydrothermal and aging degradation: A NIR spectroscopy and XRD study," Biomacromolecules 11(9), 2300-2305. DOI: $10.1021 / \mathrm{bm} 100403 \mathrm{y}$

Kol, H. S. (2010). "Characteristics of heat-treated Turkish pine and fir wood after Thermowood processing," Journal of Environmental Biology 31(6), 1007-1011. DOI: 10.2112/JCOASTRES-D-10-00093.1

Lin, Q. F. (1997). "Variation in densities and shrinkages of Chinese fir wood from plantation," Journal of Zhejiang Forestry College 14(2), 169-173.

Li, B.-G., Peng, W.-X., Qiao, J.-Z., Zhang, Z.-F., and Wu, Y.-Q. (2007). "Effect of alternate adsorption-desorption treatments on shrinkage and bulking properties of Eucalyptus urophylla $\times$ grandis," China Wood Industry 21(5), 16-17, 20.

Liu, Y. X., and Zhao, G. J. (2012). Wood Science, China Forestry Press, Beijing, China.

Love, S. A., Popov, E., Rybacki, K., Hu, X., and Salas-de la Cruz, D. (2020). "Facile treatment to fine-tune cellulose crystals in cellulose-silk biocomposites through hydrogen peroxide," International Journal of Biological Macromolecules 147, 569575. DOI: 10.1016/j.ijbiomac.2020.01.100

LY/T 2369 (2014). "Testing methods for physical and mechanical properties of desert shrub," Standardization Forestry Industry of China, Beijing, China.

Müller, U., Rätzsch, M., Schwanninger, M., Steiner, M., and Zöbl, H. (2003). "Yellowing and IR-changes of spruce wood as result of UV-irradiation," Journal of Photochemistry and Photobiology B: Biology 69(2), 97-105. DOI: 10.1016/S10111344(02)00412-8

Pandey, K. K. (1999). "A study of chemical structure of soft and hardwood and wood polymers by FTIR spectroscopy," Journal of Applied Polymer Science 71(12), 19691975. DOI:10.1002/(SICI)1097-4628(19990321)71:12<1969::AID-APP6>3.0.CO;2-D

Peng, W.-X., Zhang, Z.-F., Lu, S.-C., Liu, Q.-M., and Wu, Y.-Q. (2007). "Effects of loop drying and absorbing on wood moisture content of Eucalyptus urograndis," Journal of Liaoning Forestry Science and Technology 4, 5-8. DOI: 10.3969/j.issn.10011714.2007.04.002

Seldén, R., Nyström, B., and Långström, R. (2004). "UV aging of poly(propylene)/woodfiber composites," Polymer Composites 25(5), 543-553. DOI: 10.1002/pc.20048

Takei, T., Masashi, H., and Nobuyuki, K. (1997). "Analysis of deterioration of Horyuji old material by FT-IR," Journal of Wood Society 43(3), 285-294. DOI: 10.1080/02773819708003142

Teacă, C. A., Rosu, D., Bodîrlău, R., and Roşu, L. (2013). "Structural changes in wood under artificial UV light irradiation determined by FTIR spectroscopy and color measurements-A brief review," BioResources 8(1), 1478-1507. DOI: 10.15376/biores.8.1.1478-1507

Traoré, M., Kaal, J., and Cortizas, A. M. (2016). “Application of FTIR spectroscopy to the characterization of archeological wood," Spectrochimica Acta Part A: Molecular and Biomolecular Spectroscopy 153, 63-70. DOI: 10.1016/j.saa.2015.07.108

Wu, X.-W., Zhao, Z.-Q., Wang, X.-M., and Liu, J.-X. (2016). "Study on Salix discolor physical-mechanical properties and its variation," Wood Processing Machinery 27(2), 33-36. DOI: 10.13594/j.cnki.mcjgjx.2016.02.010 
Xia, L.-M., Qi, J.-Q., Huang, X.-Y., Xie, J.-L., Xiao, H., Luo, J.-X., Xiao, H., and Song, L. M. (2018). "Physical-mechanical properties of heartwood and sapwood in Toona sp. wood (Toona ciliata M. Roem.) before and after accelerated aging treatment," BioResources 13(4), 8409-8420. DOI: 10.15376/biores.13.4.8409-8420

Xiang-Yu, W., Guo-Dong, D., Han, G., Jin-Hu, Z., Jin-Ru, H., and Yu-Ming, Z. (2008). "Effect of zonal willow Salix psammophila checkerboard on reducing wind and stabilizing sand," Journal of Soil and Water Conservation 22(2), 42-46. DOI: 10.1016/S1872-2075(08)60071-0

Zhang, S., Ding, G.-D., Yu, M.-H., Gao, G.-L., Zhao, Y.-Y., Wang, L., and Wang, Y.-Z. (2019). "Application of boundary layer displacement thickness in wind erosion protection evaluation: Case study of a Salix psammophila sand barrier," International Journal of Environmental Research and Public Health 16(4), Article number 592. DOI: $10.3390 /$ ijerph16040592

Article submitted: March 29, 2020; Peer review completed: June 4, 2020; Revised version received and accepted: July 7, 2020; Published: July 13, 2020.

DOI: $10.15376 /$ biores. 15.3.6696-6713 